\title{
A Conversation with Mehmet Fatih Yanik
}

\author{
INTERVIEWER: SEJAL VYAS \\ Assistant Scientific Editor, Cell Reports
}

\begin{abstract}
Mehmet Fatih Yanik is a Professor of Neurotechnology at the Institute of Neuroinformatics at ETH Zürich.
\end{abstract}

Sejal Vyas: You work on neuronal circuit imaging to identify therapeutic drug combinations to correct altered brain activity patterns and neurological disorders. What led you to this approach and what was the initial inspiration for this type of research?

Dr. Yanik: My background is in engineering, computer science, and physics. One of the things that always surprised me is how we treat the brain. It is probably the most sophisticated machine we know in the universe. It is more complex than our supercomputer networks, yet when we treat brain disorders, we basically perfuse the entire brain with chemicals expecting magic to happen. And magic sometimes happens, but more often it does not. Yes, people know about the involvement of complex network dysfunctions in brain disorders and have "proposals" for treating them. Yet, there has been no demonstration for fixing brain-wide dysfunctions. My inclination was that, at least in simple animal models, we should be able to look into these circuits at a very high resolution, at a single-cell resolution over the entire brain, and test whether we can indeed fix their brain-wide network dysfunctions just like we fix man-made artificial circuits.

Sejal Vyas: It's a very high-throughput zebrafish light sheet imaging platform you're using with implanted electrodes. Were there any major technical hurdles you had to overcome to get this?

Dr. Yanik: There were a couple of challenges that we had to overcome. One of them is that we had to image neuronal activity patterns very rapidly. We developed a technique so we can scan the entire brain in about 50 milliseconds and capture activity from about 15,000 neurons in these small animals. The other challenge is that we wanted to test lots of different ideas, concepts, methods, chemicals, so we had to make the whole process fully automated by using microfluidics and robotics where we can handle and manipulate thousands of small animals very rapidly. These were some of the technical challenges. Then we had computational challenges: how to analyze the data without getting lost, how to look at it from different perspectives.

Sejal Vyas: That computation analysis side — optimizing the various things you looked at as far as how many re- gions of the brain you have to measure and how many neurons and what resolution - that all goes into huge data sets. Do you have to minimize how much you need to measure to be able to handle that?

Dr. Yanik: Those are some of the challenges. One of the most important things from our study we learned is that there's absolutely significant reason in capturing brain activity patterns at neuronal resolution. Since we were able to image these brains at single-neuron resolution, we were able to back off and increase the granularity, to reduce what we can get and make the data more coarse. Our capability to correct network dysfunctions across the entire brain, dramatically declined as we made our measurements coarser than single-neuron resolution. It sounds impossible to do such measurements in higher animal species, but I believe we are developing technologies that will make these possible even in humans.

Sejal Vyas: You used a specific zebrafish mutant that had a mutant sodium channel that has clinical implications for epilepsy. You found a two-drug polytherapy that really seemed to correct these brain activity patterns. Based on what's known about this drug combination, can you speculate on how exactly they're functioning to correct these patterns? Is it through neuronal or nonneuronal targets?

Dr. Yanik: One of the important things about our study was the approach we used. It was independent of the disease model we studied. SCN1A [sodium voltage-gated channel alpha subunit 1] causes epilepsy and autism, as you mentioned. We picked the Scnla mutant zebrafish model because it has been a predictive model and there's significant preliminary data on this model, both from our labs and also from others. SCN1A [sodium voltage-gated channel alpha subunit 1] mutations cause epilepsy and autism in humans, as you mentioned. Your question about how we identified these drug combinations... Basically, by looking at the neuronal-resolution functional connectivity patterns over the entire brain and how individual drugs perturb these connectivity patterns, we identified those drugs that perturb the brain in quite distinct means, and then we combined them to make an overall correction to the brain activity patterns that can completely normalize the dysfunction

C 2018 Yanik. This article is distributed under the terms of the Creative Commons Attribution License, which permits unrestricted reuse and redistribution provided that the original author and source are credited. 
across the entire brain. It's not clear whether we can directly take these molecules and apply them to higher animal models because these chemical combinations we identified are engineered for this particular brain network. However, our overall strategy is likely applicable to any brain because our methods do not rely on the specifics of any brain anatomy or function.

Sejal Vyas: About the difficulties in translating this to higher-order mammalian brain or having more combinations than just two drugs: Is that an area you're looking to pursue? If you were to go there, what approaches would you take?

Dr. Yanik: We learned a couple of major lessons in this study while we were trying to correct network dysfunctions in zebrafish. One of them is doing single-neuron resolution measurements was very powerful. Interestingly, we found out that we don't need to do such high-resolution measurements over the entire brain; we just need to do this over limited numbers of areas, and we also need a limited number of neurons. We don't need tens of thousands of neurons; we need maybe a few hundred neurons per area to make these kinds of network measurements sufficiently accurate to be able to subsequently correct network dysfunctions. It's one of the things that I am pushing forward in the lab, trying to develop technologies where we can make such measurements on higher animal models minimally invasively - in rodents and, hopefully, in primates one day.

Sejal Vyas: You cover other topics: One of them was focused ultrasound for drug delivery. Chronic focused ultrasound damages the blood-brain barrier, so you were trying to take a different approach to get the spatialized drug delivery. Can you talk about how your approach differs from other things that are being done in this area?

Dr. Yanik: The most obvious observation is that, when we treat brain disorders today, we perfuse the entire brain with chemicals just like we marinate meat. I think one of the fundamental issues that we need to address to treat currently intractable disorders is to be able to target circuits in a spatially specific manner. For this purpose, we have been developing a unique focused ultrasound technology. Focused ultrasound itself is something widely employed by several different labs. But what we particularly did was to use high-throughput approaches and microfluidics to design microparticles that are ultrasensitive to ultrasound waves, yet otherwise stable enough in the blood. We could then trigger these particles by focusing ultrasound anywhere in the brain to release their drug cargo without actually causing any sort of detectable brain damage. This noninvasive capability opens up immense possibilities because we can treat the brain repeatedly without harming it, which is a necessary condition for the chronic treatment of almost all brain disorders.

Our second trick was to address another major challenge: When microparticles are systemically injected into the blood, they distribute to the entire body. However, even when one targets ultrasound to a focal brain area to uncage microparticles locally, the rest of microparticles in the bloodstream eventually break down, releasing their content everywhere in the body. We developed a noninvasive approach that allows us to concentrate these microparticles in any local brain region by several orders of magnitude above their systemic levels in the body. This allows us to dramatically reduce systemic release and hence side effects of drugs while dramatically increasing their therapeutic precision and efficacy.

Finally, our technology can in principle allow locally concentrating and releasing of almost all FDA-approved neurological and neuropsychiatric drugs, making its translation to clinic more feasible.

Sejal Vyas: That sounds like a pretty powerful drug delivery mechanism for potential neurological disorders. Can that be more broadly applied to glioblastomas or other diseases of the brain that aren't necessarily neurological?

Dr. Yanik: Certainly, I think our technology can be used for many domains. Particularly in neurology though, in brain disorders, our technology can make a major difference because unlike cancer and other diseases, you often need to treat the brain chronically. We spent significant effort in making this technology minimally invasive and highly efficacious. It's ultimately going to be a very powerful technology for neurological and brain disorders. For cancers, if you are trying to locally ablate a particular area or tumor mass, our capability to concentrate drugs by physical means in very local areas after systemic injection would also be something that could potentially be very enabling by significantly reducing systemic side effects.

Sejal Vyas: What do you think is just beyond reach, that you'll be able to do in maybe another 2 to 3 years, after developing these tools?

Dr. Yanik: We are investing significant time trying to develop minimally invasive technologies for recording over multiple cortical areas in rodents and primates to estimate interareal functional connectivity using singleneuron resolution correlations as we did in zebrafish. We are working on certain neurological disorder models in the mice, and our goal in the next couple of years is to perform the kind of network measurements and manipulations we did in the zebrafish also in the rodent disorder models and to normalize their brain activity patterns to that of wild-type animals while correcting almost all abnormal behavioral phenotypical metrics.

Sejal Vyas: Neurobiology is very broad, with a lot of different approaches being taken. Do you see any way where these different approaches can inform on each other, where they can converge to look at these types of problems from different ways?

Dr. Yanik: I see a couple of major themes emerging. One of them is that the depth of our understanding of different disorders has been increasing exponentially. Neuroscientists started to pin down the mechanisms, whether addic- 
tion or autism or else, to specific circuits in the brain, to specific synaptic dysfunctions, down to specific molecules. On one hand, we are living in a highly exciting era in terms of the fundamental progress being made. On the other hand, people are also very open-minded. There are many approaches being evaluated that go far beyond the norms of existing treatments, ranging from focused ultrasound to transcranial magnetic stimulation to potential optogenetics in higher species to interrogate and manipulate brain circuits to fix disorders. If you talk about things like implanting large-scale microelectrode arrays over multiple cortical areas, it doesn't sound crazy anymore to the audience.
Sejal Vyas: Let's say tomorrow someone said, "You can no longer study anything in the brain, or neurobiology." What other areas of research would you find interesting or would want to dig into?

Dr. Yanik: I think artificial general intelligence (AGI) would be one area I would go into if I were to stop working on the brain itself. There are many commonalities between the concepts and problems we study in systems neuroscience and what the AI community is interested in. There will likely be major convergences across several domains between neuroscience and AGI in the near future. Perhaps we are all already working on AGI in one way or another. 


\section{$\$_{\text {CSH\& }}^{\infty}$ Cold Spring Harbor Symposia SYMPOSIA}

\section{A Conversation with Mehmet Fatih Yanik}

Cold Spring Harb Symp Quant Biol 2018 83: 290-292 originally published online April 23, 2019 Access the most recent version at doi:10.1101/sqb.2018.83.038018
Creative This article is distributed under the terms of the
Commons http://creativecommons.org/licenses/by/4.0/, which permits unrestricted
License reuse and redistribution provided that the original author and source are credited.

Email Alerting Receive free email alerts when new articles cite this article - sign up in Service the box at the top right corner of the article or click here. 